\title{
Approximate Closed-Form Expression of the Electric Field of a Conical Horn Antenna
}

\author{
Mohammad Asif Zaman, Md. Gaffar, Md. Mushfiqul Alam, Sayed Ashraf Mamun, \\ Sajid Muhaimin Choudhury and M. A. Matin, Member, IEEE
}

\begin{abstract}
In this paper, an approximate closed-form expression of the electric field of a conical horn with linear flare is formulated. Copolarization and cross polarization component of the electric field are calculated from the spherical field components using Ludwig's third definition of copolarization and cross polarization. The calculated field expression is found to be in close agreement with experimental results found in literature. The radiation characteristics of the conical horn are analyzed using the derived field expressions.
\end{abstract}

Index Terms-Conical horn antenna, copolarization and cross polarization.

\section{INTRODUCTION}

Horn antennas are widely used as feeds of reflectors, lenses and compound antennas [1], [2]. Their widespread use stems from their simple, solid geometry and excellent performance in terms of beam directivity.

In many applications such as satellite communication, it is required that the horn antenna handles two orthogonal polarizations and minimizes interference between them. This makes it possible to create two communication channels over the same frequency band. The conical horn antenna excited by a circular waveguide is suitable for such applications. By virtue of its axial symmetry, the conical horn antenna can handle any polarization of the exciting dominant $\mathrm{TE}_{11}$ mode [3]. Along with linear polarizations, the conical horn is also well suited for circular polarizations as well. This versatility makes the conical horn an attractive choice for feed antennas.

Theoretical studies of the radiation characteristics of a conical horn antenna requires the accurate knowledge of aperture field expressed in terms of the amplitude of modes excited at the horn [4]. Detailed analysis of the electric field by determining the modes is very complicated and requires numeric solutions. By using justified approximations, sufficiently accurate analytical solutions of the electric field of conical horns have been calculated [5], [6]. These results match closely with experimental results [7]. However, none of these field expressions are closed-form and requires numeric integration for field calculations. Evaluating numeric integrations in a computer requires more processing time compared to field calculations from a closed-form expression. Although, the time difference is small for a single design problem, it can be sufficiently large for optimization

Manuscript received July 13, 2010.

The authors are with the Department of Electrical and Electronic Engineering, Bangladesh University of Engineering and Technology, Dhaka-1000, Bangladesh. (Phone: +8801712646464; e-mail: asifzaman13@gmail.com). problems. In optimization problems, the electric field needs to be calculated at each iterative step. A typical optimization problem may take thousands of iterations. Calculating multiple numeric integrations at each step would sufficiently increase the processing time. Thus a sufficiently accurate closed-form electric field expression will be highly useful for such problems.

Although, some recent work has been done on the field properties of the conical horn [8], [9], none of the obtained results are closed-form. Some analyses have also been done on approximate gain formulas of conical horns [10], [11]. These works also lack complete electric field analysis.

A novel approximate closed form expression of the electric field of a conical horn antenna is presented in this paper. The derived results are verified by comparing it with experimental data found in literature. Using the derived expressions, copolarization and cross polarization components of the electric field are computed. The calculations of these polarization components are necessary because, experimental measurements are polarization dependent [12]. Therefore, in order to compare with experimental results, polarization dependent electric field expressions are required. Finally, using the derived expressions, the two dimensional and three dimensional radiation characteristics of the horn are analyzed.

\section{THEORY}

The geometry of conical horn antenna with linear flare is shown in Fig. 1. The coordinate system in relation to the geometry is also shown. Here $a_{w}$ is the waveguide radius, $a$ is the aperture radius, $L$ is the length of the horn, $h$ is the length of the flare section, and $\theta_{o}$ is the semi flare angle.

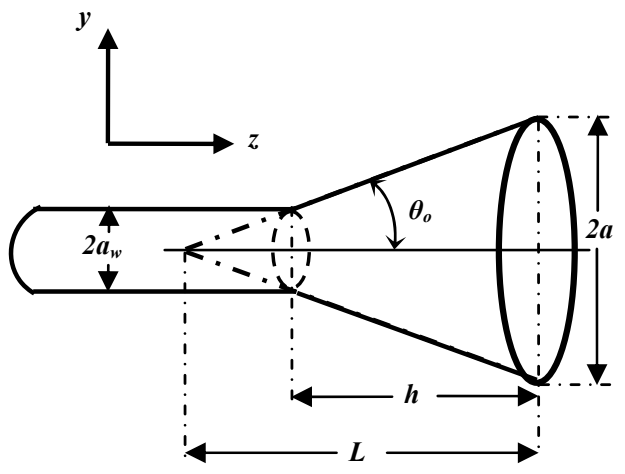

Fig. 1 Geometry of a conical horn antenna with linear flare. 


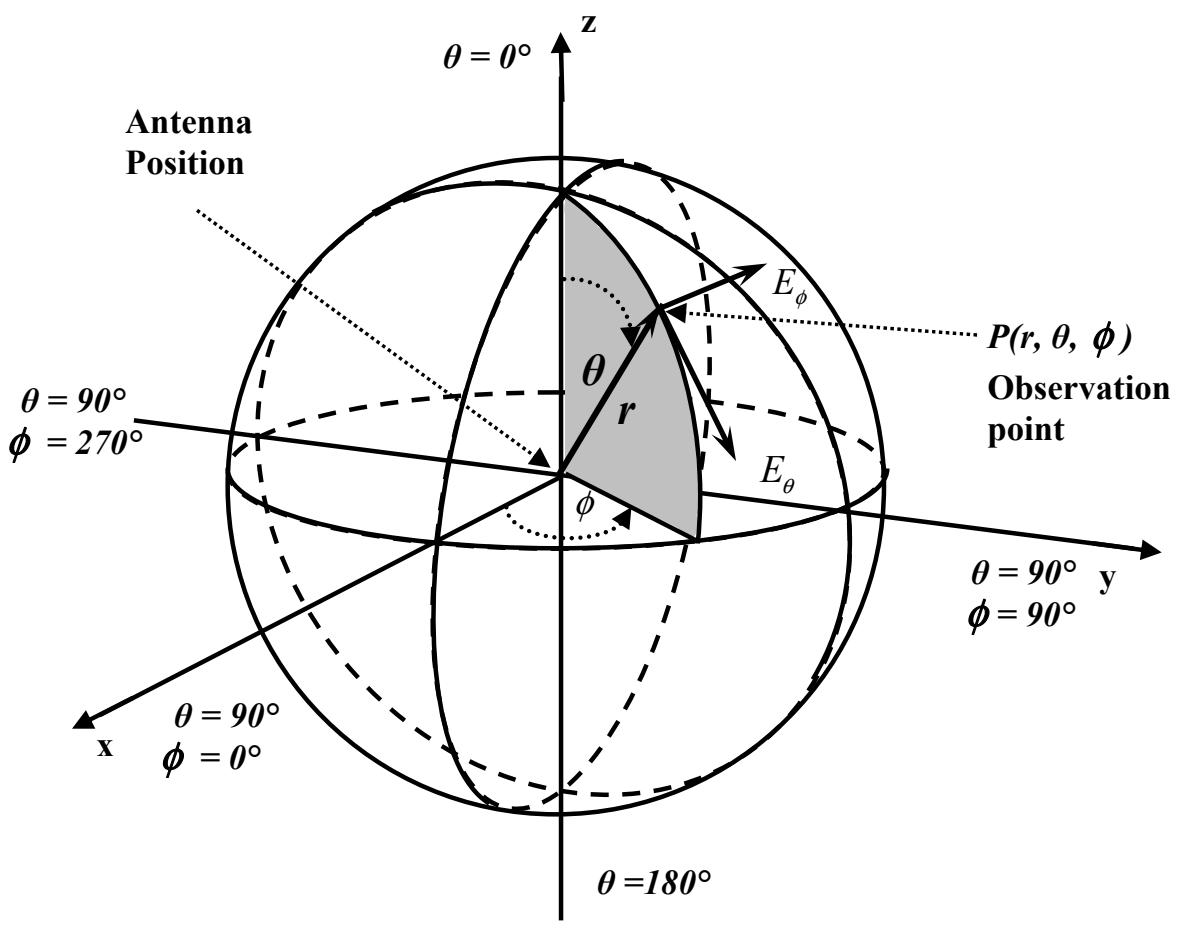

Fig. 2 Three dimensional coordinate system showing the electric field components.

The radiation characteristic of the conical horn antenna depends on the dimension parameters. The dimension parameters determine the modes generated in the horn. The modes in an infinite conical horn can be expressed exactly in terms of a combination of spherical Hankel and Legendre functions [3]. Numerical methods such as mode matching, finite element and finite difference time domain can be used to find the electric field radiated by the horn. However, sufficiently accurate results can be obtained from a dominant mode approximation analysis. For a conical horn excited in the dominant $\mathrm{TE}_{11}$ mode, the electric field can be calculated using vector diffraction formula [4]. Assuming quadratic phase approximation, the field radiated by the horn for the dominant mode has been determined by Narasimhan and Rao and is given by [3], [4]:

$E_{\theta}(r, \theta, \phi)=\frac{j E_{o} k a^{2}}{2} \frac{e^{-j k r}}{r}\left[G_{0}(w)-G_{2}(w)\right] \cos \phi$

$E_{\phi}(r, \theta, \phi)=-\frac{j E_{o} k a^{2}}{2} \frac{e^{-j k r}}{r} \cos \theta\left[G_{0}(w)-G_{2}(w)\right] \sin \phi$.

Where,

$$
\begin{aligned}
G_{m}(w)=\frac{1}{a^{2}} \int_{0}^{a} J_{m}\left(p_{11} \rho^{\prime} / a\right) J_{m}\left(w \rho^{\prime}\right) \\
\\
\quad \times \exp \left(-j k \rho^{\prime 2} / 2 L\right) \rho^{\prime} d \rho^{\prime} .
\end{aligned}
$$

Here,

$(r, \theta, \phi)=$ spherical coordinates of the observation point,

$E_{\theta}, E_{\phi}=$ spherical components of the electric field,

$E_{o}=$ Amplitude of the dominant $\mathrm{TE}_{11}$ mode,

$k=$ wave number $=2 \pi / \lambda$,

$\lambda=$ wavelength,

$a, L=$ dimension parameters of the horn,

$w=k \sin \theta=$ angular coordinate,

$p_{11}=1.841184=$ first non-vanishing root of $J_{1}^{\prime}($.$) ,$

$\rho^{\prime}=$ variable of integration, and
$J_{m}()=$. Bessel function of the first kind and order $m$.

The three dimensional spherical coordinate system indicating antenna position, observation point and electric field component is shown in Fig. 2.

The analysis provided in this paper starts with (1), (2) and (3). The equations are accepted to be accurate as they match closely with experimental results. The results are accurate for semi-flare angle less than $35^{\circ}$ for medium to long horns [3]. The integration in (3) for $m=0$ and 2 must be evaluated to find the electric field components. As these integrations do not have exact closed-form solutions, the resulting electric field expressions are not closed-form. In this paper, an approximate closed-form solution of (3) is derived for $m=0$ and 2. For ease of calculation, (3) is modified by introducing a new variable $x=w \rho^{\prime}$. Substituting this value in (3) gives:

$$
G_{m}(w)=\frac{1}{(w a)^{2}} \int_{0}^{w a} J_{m}\left(x p_{11} / w a\right) J_{m}(x) e^{-j n x^{2}} x d x .
$$

Here, $n=k / 2 L w^{2}$.

Approximate expressing of $G_{0}(w)$ and $G_{2}(w)$ are calculated from (4). The results are used to find the closed-form expression of the electric field components described by (1) and (2).

The copolarization and the cross polarization component of the electric field are computed from the spherical field components using Ludwig's third definition of cross polarization [13]. The relation is given by [3]:

$$
\left[\begin{array}{c}
E_{c o} \\
E_{x p}
\end{array}\right]=\left[\begin{array}{cc}
\cos \left(\phi-\phi_{o}\right) & -\sin \left(\phi-\phi_{o}\right) \\
-\sin \left(\phi-\phi_{o}\right) & -\cos \left(\phi-\phi_{o}\right)
\end{array}\right] \cdot\left[\begin{array}{c}
E_{\theta} \\
E_{\phi}
\end{array}\right] \text {. }
$$

Here,

$E_{c o}=$ copolarization component of the electric field,

$E_{x p}=$ cross polarization component of the electric field, and $\phi_{o}=$ reference polarization direction taken at $\mathrm{x}$ axis. 
Copolarization refers to the polarization in the desired direction and cross polarization refers to the undesired polarization located at $\pm 90^{\circ}$ from the copolarization direction [12]. Cross polarization indicates the interference created by the horn when two orthogonal polarizations are transmitted/received at the same time.

\section{EVALUATION OF $\mathrm{G}_{0}$ INTEGRATION}

Calculating the electric field using (1) and (2) requires evaluation of two integrations. This first integration, $G_{0}(w)$ is found by putting $m=0$ in (4):

$$
G_{0}(w)=\frac{1}{(w a)^{2}} \int_{0}^{w a} J_{0}\left(x p_{11} / w a\right) J_{0}(x) e^{-j n x^{2}} x d x .
$$

There is no exact closed-form solution of this integration. If one of the Bessel functions is replaced by an approximate function, the integration can have an approximate closed-form solution. It is noted that the argument of the first Bessel function varies from 0 to $p_{11}=1.841184$ over the integration limit of $x=0$ to $w a$. An appropriate quadratic function can be used to model the Bessel function over this limit. For ease of integration, it is required that the modeled quadratic function do not contain any terms with odd powers of its argument. Through simple mathematical analysis, the modeled function, $f_{0}(x)$ is found to be:

$$
f_{0}(x)=1-0.683\left(\frac{x}{w a}\right)^{2} \approx J_{0}\left(x p_{11} / w a\right) .
$$

The modeled quadratic function along with the actual Bessel function is shown in Fig. 3. It is clear that the modeled function follows the original function very closely over the integration interval. Substituting (7) in (6):

$$
\begin{aligned}
G_{0}(w)=\frac{1}{(w a)^{2}} \int_{0}^{w a} x J_{0}(x) e^{-j n x^{2}} d x \\
-\frac{0.683}{(w a)^{4}} \int_{0}^{w a} x^{3} J_{0}(x) e^{-j n x^{2}} d x .
\end{aligned}
$$

$$
\text { or, } G_{0}(w)=G_{01}(w)-G_{02}(w) \text {. }
$$

The two integrations must be performed separately. The first integration is given by:

$$
G_{01}(w)=\frac{1}{(w a)^{2}}\left[I_{1}\right]_{0}^{w a} .
$$

Where,

$$
I_{1}=\int x J_{0}(x) e^{-j n x^{2}} d x .
$$

Integrating by parts [14], [15]:

$$
\begin{array}{r}
I_{1}=e^{-j n x^{2}} \int x J_{0}(x) d x-\int\left\{\frac{d}{d x} e^{-j n x^{2}} \int x J_{0}(x) d x\right\} d x \\
=x J_{1}(x) e^{-j n x^{2}}+(j 2 n) \int x^{2} J_{1}(x) e^{-j n x^{2}} d x .
\end{array}
$$

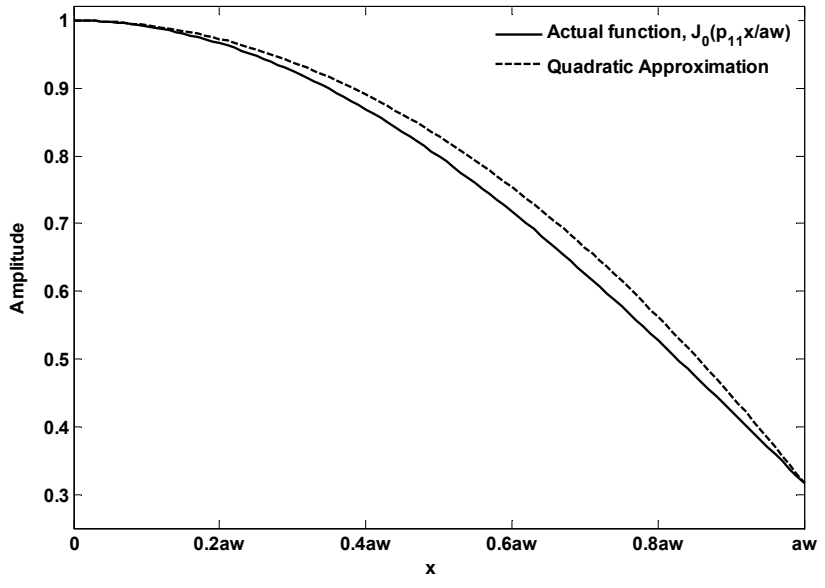

Fig. 3 Quadratic approximation of the Bessel function $J_{0}($.).

The following property of Bessel functions with $p=0$ is used for the integration [1]:

$$
\int x^{p+1} J_{p}(x) d x=x^{p+1} J_{p+1}(x)
$$

Integrating by parts again, (12) becomes,

$$
\begin{aligned}
I_{1}=x J_{1}(x) e^{-j n x^{2}} & +(j 2 n) x^{2} J_{2}(x) e^{-j n x^{2}} \\
& +(j 2 n)^{2} \int x^{3} J_{2}(x) e^{-j n x^{2}} d x .
\end{aligned}
$$

Continuing this process gives the result of the integration in terms of an infinite series:

$$
I_{1}=e^{-j n x^{2}} \sum_{l=1}^{\infty} x^{l} J_{l}(x)(j 2 n)^{l-1} .
$$

Substituting (15) in (10):

$$
G_{01}(w)=\frac{e^{-j n(w a)^{2}}}{w a} \sum_{l=1}^{\infty}(j 2 n w a)^{l-1} J_{l}(w a) .
$$

As higher order Bessel functions damp out much more rapidly, truncating the infinite series of (16) still gives sufficiently accurate results. It has been found that taking 6 terms makes this result very close to results found from numeric integration.

Another similar integration must be performed to formulate $G_{02}(w)$. The second integration of $(8)$ is given by:

$$
G_{02}(w)=\frac{0.683}{(w a)^{4}}\left[I_{2}\right]_{0}^{w a} .
$$

Where,

$$
I_{2}=\int x^{3} J_{0}(x) e^{-j n x^{2}} d x .
$$

Integrating by parts:

$$
\begin{aligned}
I_{2} & =e^{-j n x^{2}} \int x^{3} J_{0}(x) d x-\int\left\{\frac{d}{d x} e^{-j n x^{2}} \int x^{3} J_{0}(x) d x\right\} d x \\
& =e^{-j n x^{2}} Z_{0}(x)+(j 2 n) \int x Z_{0}(x) e^{-j n x^{2}} d x .
\end{aligned}
$$




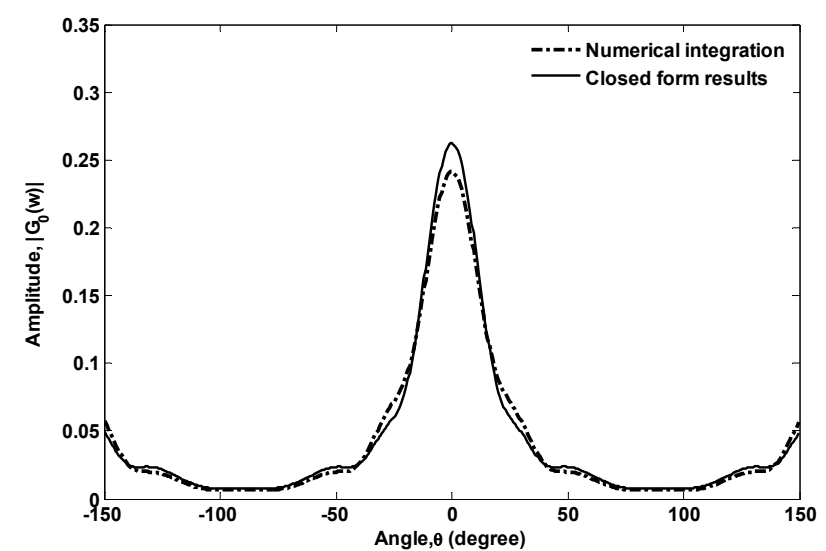

Fig. 4 Comparison of numeric integration results and the derived closed-form expression of $G_{0}(w)$ for $a=1.7 \lambda$ and $L=3.5 \lambda$. [1]:

The following property of Bessel functions is used here

$$
\int x^{3} J_{0}(x) d x=x^{3} J_{1}(x)-2 x^{2} J_{2}(x)=Z_{0}(x)
$$

Continuing the integration of (19):

$$
\begin{aligned}
I_{2}=e^{-j n x^{2}} Z_{0}(x) & +(j 2 n) e^{-j n x^{2}} Z_{1}(x) \\
+ & (j 2 n)^{2} \int x Z_{1}(x) e^{-j n x^{2}} d x .
\end{aligned}
$$

Where,

$$
\int x Z_{0}(x) d x=x^{4} J_{2}(x)-4 x^{3} J_{3}(x)=Z_{1}(x) \text {. }
$$

Continuing this process results in the infinite series solution of the integrations given by:

$$
I_{2}=x^{3} e^{-j n x^{2}} \sum_{l=1}^{\infty}(j 2 n x)^{l-1}\left\{J_{l}(x)-\frac{2 l}{x} J_{l+1}(x)\right\} .
$$

Substituting (23) into (17):

$$
\begin{aligned}
G_{02}(w)=0.683 \frac{e^{-j n(w a)^{2}}}{w a} \sum_{l=1}^{\infty} & (j 2 n w a)^{l-1} \\
& \times\left\{J_{l}(w a)-\frac{2 l}{w a} J_{l+1}(w a)\right\} .
\end{aligned}
$$

It is found that like (16), only the first 6 terms of (24) is sufficient to give accurate results. By substitution (16) and (24) in (9) and truncating the summation to the first six terms for both cases, the following results are obtained:

$$
\begin{aligned}
G_{0}(w)=\frac{e^{-j n(w a)^{2}}}{w a} & \sum_{l=1}^{6}(j 2 n w a)^{l-1} \\
& \times\left\{0.317 J_{l}(w a)+\frac{1.366 l}{w a} J_{l+1}(w a)\right\} .
\end{aligned}
$$

The accuracy of (25) is verified by comparing it with numeric integration results for a particular set of dimension parameters of the horn. The comparison is shown in Fig. 4. The independent variable is changed from the $w$ to $\theta$ by using relation $\theta=\sin ^{-1}(w / k)$. It is clear that the closed form

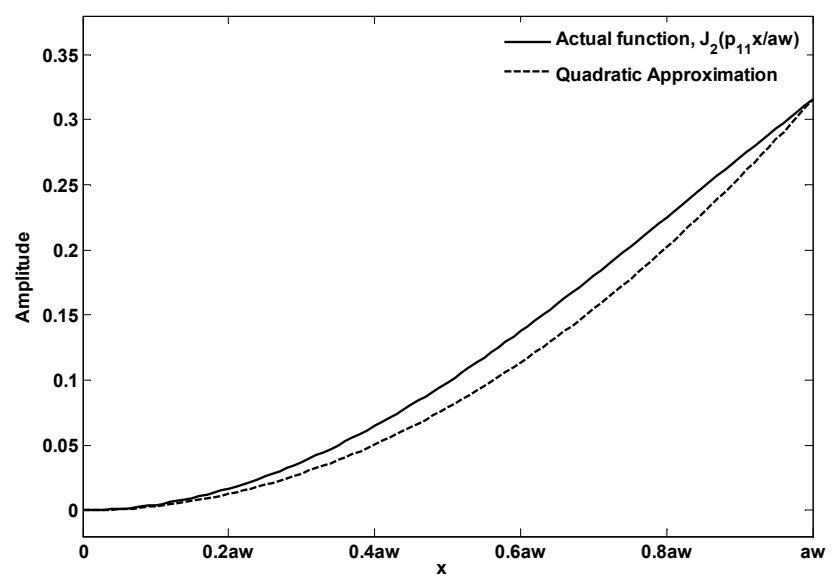

Fig. 5 Quadratic approximation of the Bessel function $J_{2}($.).

expression matches very closely with the numeric integration results. Equation (25) is also found to be sufficiently accurate for any arbitrary practical dimension parameters of the conical horn.

\section{Evaluation OF $\mathrm{G}_{2}$ INTEGRATION}

The integration $G_{2}(w)$ must also be calculated to formulate the electric field. The integration $G_{2}(w)$ can be found by putting $m=2$ in (4):

$$
G_{2}(w)=\frac{1}{(w a)^{2}} \int_{0}^{w a} J_{2}\left(x p_{11} / w a\right) J_{2}(x) e^{-j n x^{2}} x d x .
$$

This integration is performed using a strategy similar to the one used for calculating $\mathrm{G}_{0}(w)$. The first second order Bessel function is replaced with an approximate quadratic function. The approximate function is formulated under the conditions that it must closely resemble the second order Bessel function and it must not contain any terms with odd powers of $x$. The modeled quadratic function is given by:

$$
f_{2}(x)=0.3157\left(\frac{x}{w a}\right)^{2} \approx J_{2}\left(x p_{11} / w a\right) .
$$

The quadratic approximate function and the actual Bessel function are shown in Fig. 5. It can be clearly seen that the modeled function closely resembles the Bessel function. Now, substituting (27) in (26):

$$
\begin{aligned}
& G_{2}(w)=\frac{0.3157}{(w a)^{4}} \int_{0}^{w a} x^{3} J_{2}(x) e^{-j n x^{2}} d x \\
& \text { or, } G_{2}(w)=\frac{0.3157}{(w a)^{4}}\left[I_{3}\right]_{0}^{w a} .
\end{aligned}
$$

Where,

$$
I_{3}=\int x^{3} J_{2}(x) e^{-j n x^{2}} d x .
$$

Integrating by parts:

$$
I_{3}=e^{-j n x^{2}} \int x^{3} J_{2}(x) d x-\int\left\{\frac{d}{d x} e^{-j n x^{2}} \int x^{3} J_{2}(x) d x\right\} d x
$$




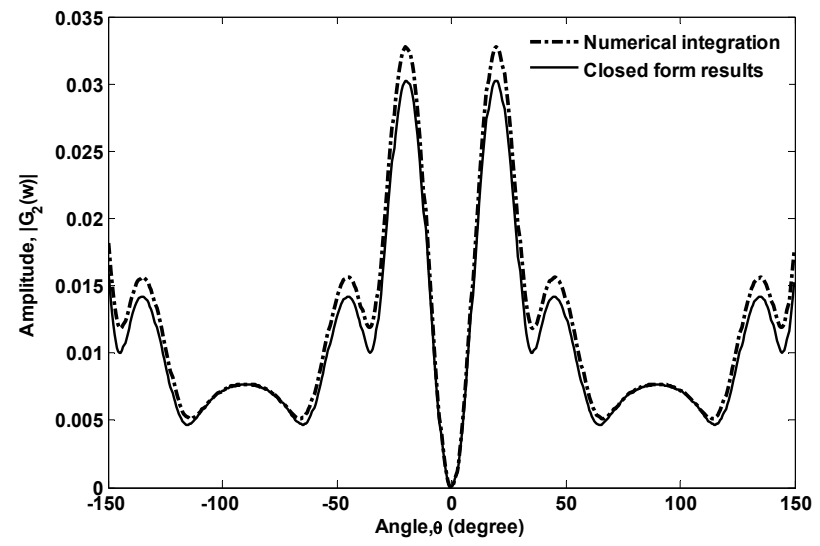

Fig. 6 Comparison of numeric integration results and the derived closed-form expression of $G_{2}(w)$ for $a=1.7 \lambda$ and $L=3.5 \lambda$.

$$
\text { or, } I_{3}=x^{3} J_{3}(x) e^{-j n x^{2}}+(j 2 n) \int x^{4} J_{3}(x) e^{-j n x^{2}} d x \text {. }
$$

Equation (13) is used with $p=3$ for this integration. Continuing the integration of (30) gives an infinite series result expressed as:

$$
I_{3}=e^{-j n x^{2}} \sum_{l=1}^{\infty} x^{l+2} J_{l+2}(x)(j 2 n)^{l-1} .
$$

It is found that taking the first 6 terms of summation of (31) gives sufficiently accurate results. Substituting (31) in (28) and truncating the summation to the first 6 terms gives:

$$
G_{2}(w)=0.3157 \frac{e^{-j n(w a)^{2}}}{w a} \sum_{l=1}^{6}(j 2 n w a)^{l-1} J_{l+2}(w a) .
$$

Equation (32) is verified by comparing it with numerical integration results. The comparison is shown in Fig. 6 for a particular set of dimension parameters. It is evident that the closed-form expression is sufficiently accurate. Equation (32) is also tested for other arbitrary practical dimension parameters and found to be accurate.

\section{ELECTRIC FIELD FORMULATION}

As both $G_{0}(w)$ and $G_{2}(w)$ have been calculated, the electric field components can easily be formulated. Substituting (25) and (32) in (1) gives:

$$
\begin{array}{r}
E_{\theta}(r, \theta, \phi)=\frac{j E_{o} k a^{2}}{2} \frac{e^{-j k r}}{r} \cos \phi \sum_{l=1}^{6}\left[(j 2 n w a)^{l-1}\right. \\
\times\left\{0.317 J_{l}(w a)+\frac{1.366 l}{w a} J_{l+1}(w a)\right. \\
\left.\left.-0.3157 J_{l+2}(w a)\right\}\right] .
\end{array}
$$

Similarly, substituting (25) and (32) in (2) gives:

$$
\begin{array}{r}
E_{\phi}(r, \theta, \phi)=-\frac{j E_{o} k a^{2}}{2} \frac{e^{-j k r}}{r} \sin \phi \cos \theta \sum_{l=1}^{6}\left[(j 2 n w a)^{l-1}\right. \\
\times\left\{0.317 J_{l}(w a)+\frac{1.366 l}{w a} J_{l+1}(w a)\right. \\
\left.\left.+0.3157 J_{l+2}(w a)\right\}\right] .
\end{array}
$$

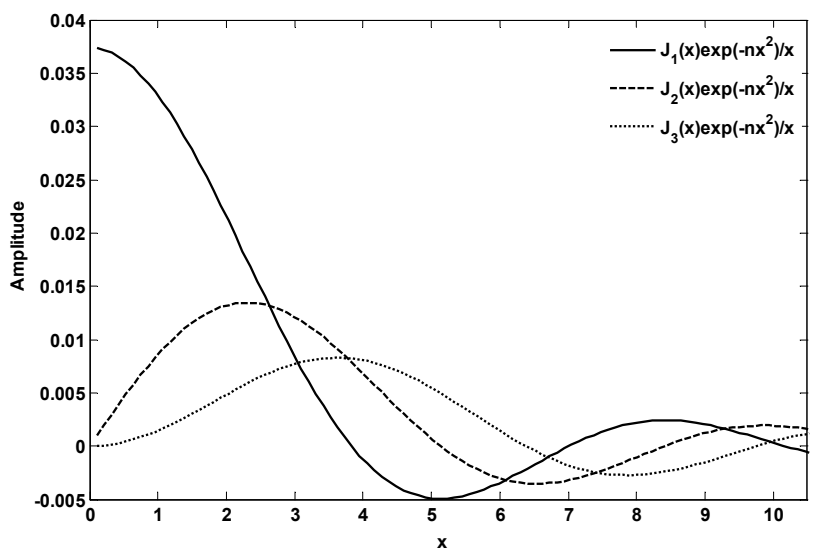

Fig. 7 Comparison of decrease rate of higher order Bessel function terms.

Equation (33) and (34) are approximate closed-form expressions of the radiated electric field of the conical horn antenna.

Fig. 7 shows the rapid decrease of higher order Bessel function terms. This illustrates the justification of truncating the infinite series solution of $G_{0}(w)$ and $G_{2}(w)$.

To compare the derived results with experimental data, it is necessary to formulate the copolarization and cross polarization components of the electric field. This can be accomplished by substituting (33) and (34) in (5). The copolarization component is usually expressed as a function of $\theta$ at a constant $\phi$ plane. Measurements are done in the two principle planes: E-plane $\left(\phi=0^{\circ}\right)$ and H-plane $\left(\phi=90^{\circ}\right)$ in most experiments. The copolarization component of the electric field at $\phi=0^{\circ}$ plane is found from (5) as:

$$
\left.E_{c o}\right|_{\phi=0^{\circ}}=\left.E_{\theta}\right|_{\phi=0^{\circ}} \text {. }
$$

The reference polarization angle, $\phi_{o}$ is taken as $0^{\circ}$. The copolarization component at $\phi=90^{\circ}$ can be similarly found from (5) as:

$$
\left.E_{c o}\right|_{\phi=90^{\circ}}=-\left.E_{\phi}\right|_{\phi=90^{\circ}} .
$$

Equation (35) and (36) can easily be evaluated by using (33) and (34).

Cross polarization is usually expressed as a function $\theta$ of at $\phi=45^{\circ}$ plane. This is done because cross polarization is maximum at this plane. Again, using (5), the cross polarization is expressed as:

$$
\left.E_{x p}\right|_{\phi=45^{\circ}}=-\frac{1}{\sqrt{2}}\left[E_{\theta}+E_{\phi}\right]_{\phi=45^{\circ}} .
$$

For comparison purposes, copolarization and cross polarization are often shown in the same plot. The graphs are normalized with respect to the maximum value of the copolarization amplitude.

The electric field in the far field or Fraunhofer region of an antenna is independent of the distance of the observation point from the antenna [1], [2]. Most analysis of radiation characteristics of an antenna involves the Fraunhofer zone. 


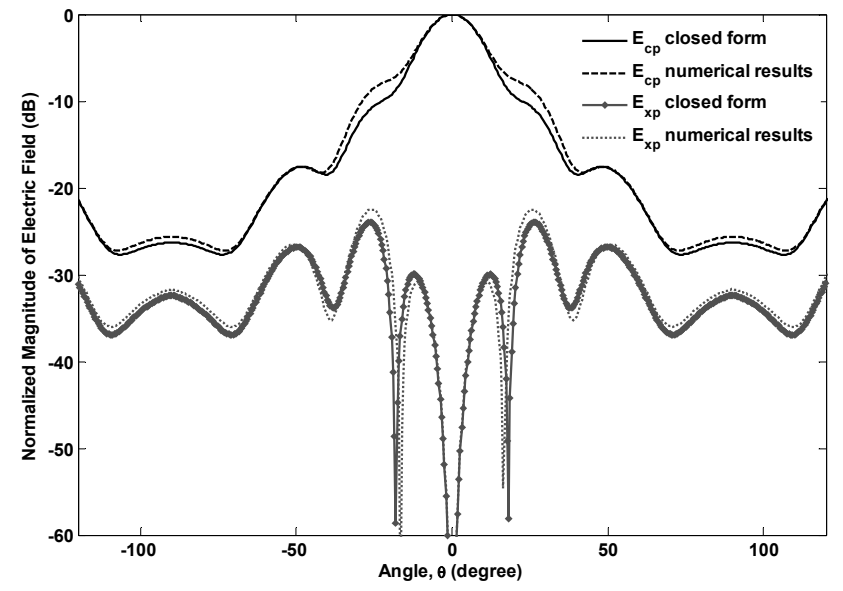

Fig. 8 Copolarization electric field $\left(\phi=0^{\circ}\right)$ and cross polarization electric field $\left(\phi=45^{\circ}\right)$ of a conical horn with $a=1.7 \lambda$ and $L=3.5 \lambda$.

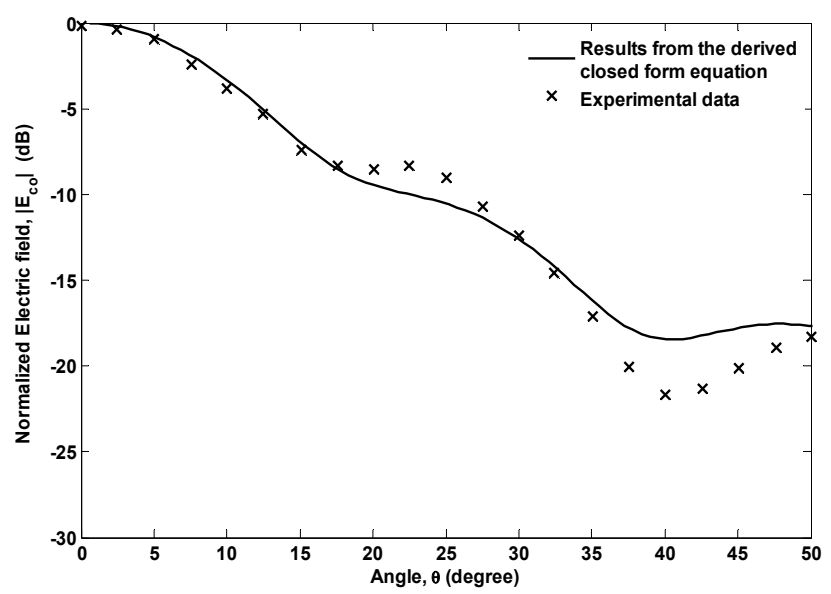

Fig. 10 Comparison of copolarization electric field at $\phi=0^{\circ}$ plane calculated from the closed-form expression and experimental data for a conical horn with $a=1.7 \lambda$ and $L=3.5 \lambda$.

Antenna measurements are also performed on the Fraunhofer zone. So, to compare with experimental data, only the angular variation of the electric field is considered. The terms containing the distance variable $r$, are ignored.

Fig. 8 shows the copolar and cross polar components of the electric field of a conical horn antenna in the Fraunhofer zone. Typical horn dimensions are assumed. The copolar component is calculated for $\phi=0^{\circ}$ plane using (35) and the cross polar component is calculated for $\phi=45^{\circ}$ plane using (37). The results are normalized with respect to the maximum value of the copolar component. To verify the accuracy of the derived closed-form expressions, numerical integration results are also shown in the same graph. It is observed that the derived closed-form expressions match closely with numerical integration results for both copolarization and cross polarization electric field components. The expressions are also found to be accurate for other $\phi$ planes and other practical dimension parameters. It is seen that the maximum cross polarization level of the conical horn antenna is approximately $-23 \mathrm{~dB}$. Corrugated horn antennas have a much lower cross polarization level [16]. This makes them suitable as feeds for parabolic or

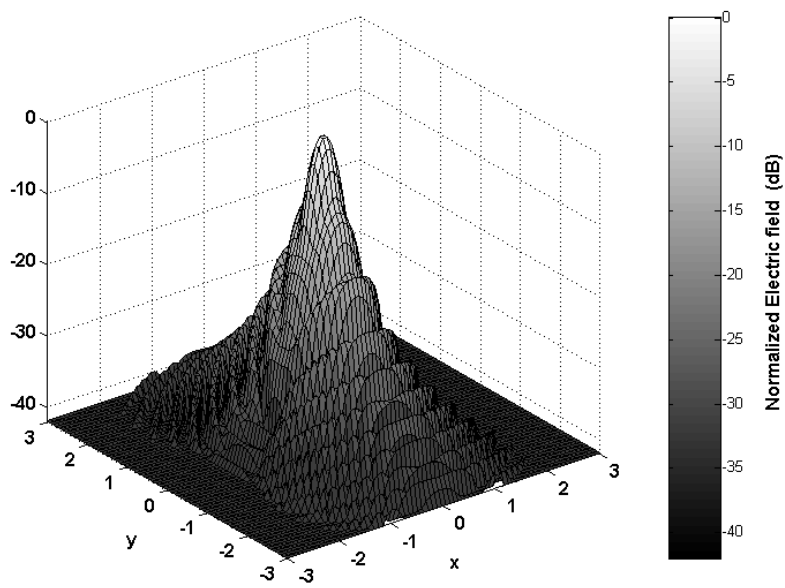

Fig. 9 Three dimensional radiated electric field of a conical horn with dimension parameters $a=1.7 \lambda$ and $L=3.5 \lambda$.

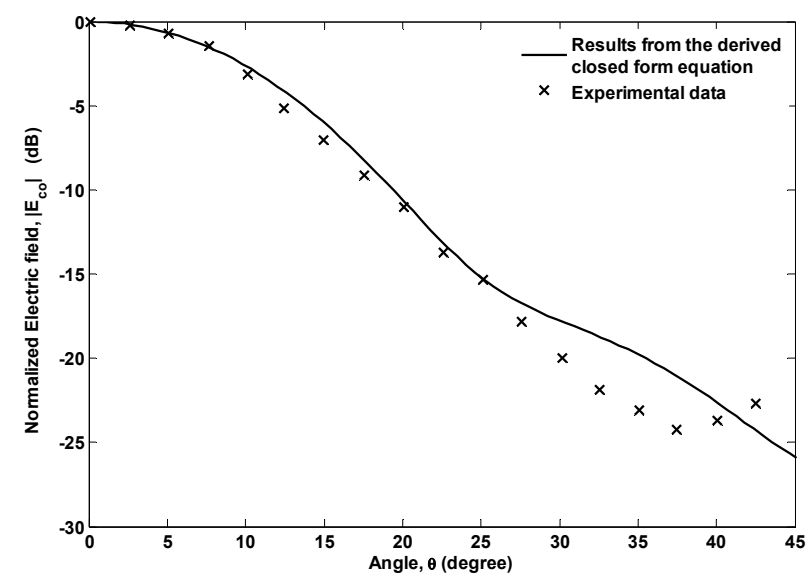

Fig. 11 Comparison of copolarization electric field at $\phi=90^{\circ}$ plane calculated from the closed-form expression and experimental data for a conical horn with $a=1.7 \lambda$ and $L=3.5 \lambda$.

Cassegrain reflectors [17]. However, the conical horn is much simpler and cheaper to construct because of its simple geometry. In many applications, the performance of the conical horn is acceptable. In such cases, the conical horn is preferred to the corrugated horn [18].

Fig. 9 shows the three dimensional normalized copolar electric field component in the Fraunhofer region. Equation (33) and (34) are used to generate the three dimensional plot. The main lobe of the horn antenna along with the side lobes is clearly visible in the figure.

Fig. 10 and Fig. 11 show comparisons of the derived closed-form expressions with experimental data. The experimental data are obtained from literature [3], [7]. The copolarization electric field in the Fraunhofer region is calculated using (35) and (36) along with (33) and (34). It can be observed that the derived closed-form expressions are in close agreement with the experimental results. This verifies the mathematical derivations presented in this paper.

It is found that when formulating the electric field using computer simulation, the computational time is much less if the closed-form expressions are used. The numerical integrations require more time to compute. As the derived closed-form expressions are sufficiently accurate for most engineering applications, they can be used for design and optimization problems to reduce computational time. 


\section{CONCLUSION}

A novel approximate closed-form expression of electric field of a conical horn is presented here. The detailed mathematical analysis used to derive the electric field components are presented in the paper. The expressions are compared with experimental data and found to be in excellent agreement. The derived expressions can be highly useful for design and optimization problems as they will significantly reduce the computational time.

\section{REFERENCES}

[1] Constantine A. Balanis, Antenna Theory Analysis and Design, $3^{\text {rd }}$ ed., John Wiley \& Sons, 2005.

[2] John D. Kraus, Ronald J. Marhefka, and Ahmad S. Khan, Antennas for all applications, $3^{\text {rd }}$ ed., Tata McGraw-Hill, 2007.

[3] Dr. John L Volakis, Trevor S. Bird and Allan W. Love, Antenna Engineering Handbook, Chapter 14: Horn Antennas, $4^{\text {th }}$ edition, McGraw-Hill, 2007.

[4] M. S. Narasimhan, B. V. Rao, "Modes in a conical horn: new approach," Proceedings of the Institution of Electrical Engineers, vol.118, no.2, Feb. 1971, pp. 287-292.

[5] M. G. Schorr, F. J. Beck, "Electromagnetic field of the conical horn," Journal of Applied Physics, vol.21, no.8, Aug. 1950, pp. 795-801.

[6] M. Narasimhan, B. Rao, "Radiation from conical horns with large flare angles," IEEE Trans. Antennas Propagat., vol.19, no.5, Sept. 1971, pp. 678-681.

[7] A. P. King, "The radiation characteristics of conical horn antennas," Proceedings of the IRE, vol.38, no.3, Mar. 1950, pp. 249-251.

[8] P. K. Dube, S. P. Singh, "Far-field patterns of metal and dielectric-wall conical horn antennas," Microwave and Optical Technology Letters, vol. 36, no. 6, 2003, pp. 447-449.

[9] J. Bornemann, "Modified analysis of conical horns," Microwave and Optical Technology Letters, vol. 10, no. 2, 1995, pp. 91-94.

[10] C. Y. Tan, K. T. Selvan, "An improved approximate gain formula for conical horn," Microwave and Optical Technology Letters, vol. 49, no. 4, 2007, pp. 971-973.

[11] R. C. Hansen, "On the directivity of conical horn antennas," Microwave and Optical Technology Letters, vol. 1, no. 10, 1988, pp. 386-388.

[12] Constantine A. Balanis, Ed., Modern Antenna Handbook. John Wiley \& Sons, 2008.

[13] A. Ludwig, "The definition of cross polarization," IEEE Trans. Antennas Propagat., vol.21, no.1, Jan. 1973, pp. 116-119.

[14] David V. Widder, Advanced Calculus, $2^{\text {nd }}$ ed., Prentice-Hall, 1961

[15] Tom M. Apostol, Calculus Volume II, $2^{\text {rd }}$ ed., John Wiley \& Sons, 1969.

[16] M. A. Matin, M. A. Zaman, S. M. Choudhury, M. Gaffar, "Analysis of a conical corrugated horn operating in the K-band with low cross-polarization and high aperture efficiency, and observing its radiation patterns," Antennas and Propagation Society International Symposium, APSURSI, 1-5 June 2009, pp. 1-4.

[17] M. A. Zaman, S. M. Choudhury, M. Gaffar, M. A. Matin, "Modeling the illumination function of a cassegrain reflector for a corrugated horn feed and calculation of the far field pattern," Loughborough Antennas \& Propagation Conference, LAPC, 16-17 Nov. 2009, pp. 101-104.

[18] Kwok Kee Chan, S. K. Rao, "Design of high efficiency circular horn feeds for multibeam reflector applications," IEEE Trans. Antennas Propagat., vol.56, no.1, Jan. 2008, pp. 253-258. 